\title{
Personal Goals of Women Recently Diagnosed with Breast Cancer: Protocol for a Cohort Study
}

\author{
Andrea Janette Chow $^{1}$, Dean Anthony Fergusson ${ }^{1,2}$, Dugald Seely ${ }^{2,3}$, Sarah Young ${ }^{3}$, Anne Pitman ${ }^{3}$, Julie
} Ennis $^{3}$, Sarah Asad ${ }^{2}$, Justin Presseau ${ }^{1,2}$

${ }^{1}$ Faculty of Medicine, University of Ottawa

2 Ottawa Hospital Research Institute (OHRI), Ottawa

${ }^{3}$ Ottawa Integrative Cancer Centre, Ottawa

ABSTRACT

Objectives: This study aims to identify the personal goals of women with breast cancer, to describe the characteristics of participants' personal goals over four months, and to identify barriers and facilitators to their pursuit.

Methods: This protocol outlines plans to conduct a prospective cohort study. We will recruit women participating in the Ottawa Integrative Cancer Centre's Head Start program (an integrative oncology psychoeducational program in Ottawa, Canada), and those on the program's waiting list if possible. We anticipate a sample size of approximately 18 to 36 women. Prior to the beginning of Head Start, participants will identify their current personal goals and rate them on various dimensions on a questionnaire. At one and three months, participants will re-assess their goals and their goal pursuit. In a one-on-one interview at three months, they will identify barriers and facilitators to the pursuit of their goals. We will analyze quantitative data using descriptive and inferential statistics, and qualitative data using thematic content analysis.

Conclusion: Findings from this study will identify important information about the personal goals of women recently diagnosed with breast cancer that can help to support the process of positive goal adjustment and enhance support to these women.

\section{RÉSUMÉ}

Objectifs : Cette étude vise à identifier les objectifs personnels des femmes atteintes d'un cancer du sein, à décrire les caractéristiques des objectifs personnels des participantes sur une période de quatre mois, et à identifier les obstacles et les facilitateurs à leur poursuite.

Méthodes : Ce protocole décrit les plans pour mener une étude de cohorte prospective. Nous recruterons des femmes qui participeront au programme Head Start du Centre de cancérologie intégrative d'Ottawa (un programme psychopédagogique intégratif en oncologie à Ottawa, au Canada) et celles qui sont sur la liste d'attente du programme, si possible. Nous prévoyons un échantillon d'environ 18 à 36 femmes. Avant le début de Head Start, les participantes identifieront leurs objectifs personnels actuels et les noteront sur différentes dimensions dans un questionnaire. À un et trois mois, les participantes réévalueront leurs objectifs et la poursuite de leur objectif. Dans une entrevue individuelle à trois mois, elles identifieront les obstacles et les facilitateurs à la poursuite de leurs objectifs. Nous analyserons les données quantitatives à l'aide de statistiques descriptives et inférentielles, et les données qualitatives à l'aide d'analyses de contenu thématiques.

Conclusion : Les résultats de cette étude permettront d'identifier des informations importantes sur les objectifs personnels des femmes récemment diagnostiquées avec un cancer du sein qui peuvent aider à soutenir le processus d'ajustement positif des objectifs et améliorer le soutien à ces femmes.

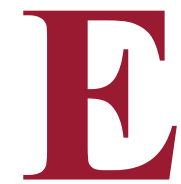
veryday life is characterized by the pursuit of multiple personal goals, such as learning to salsa dance or spending more time with family, which together describe important expressions of life motivations [1]. A personal goal is an individual's cognitive expression of a desired state or process which provides directional motivation towards that state; it defines what individuals do and strive for in everyday life $[2,3]$. Goals may be described at various levels of aggregation [2]. For example, personal strivings are mid-lev- el goals, which often become the inputs of higher-order goals and life meaning $[2,4]$. Trying to always say hello to the coffee shop clerk facilitates the higher-order goal of being a good person. Personal projects, a closely related term, are also mid-level, individually defined, characterized by action and shaped by an individual's environment [5-7]. For consistency, we use the term personal goals in the sense of personal projects and personal strivings. Goal-setting theory suggests that conscious goalsetting and motivation is important in determining individual

Keywords: Breast cancer; Personal goals; Goal pursuit; Integrative oncology; Observational study 
performance and satisfaction [8]. Diagnosis with a serious illness such as cancer, however, can affect an individual's day-today physical resources and time, thus disrupting the pursuit of personal goals [9-11]. Among women with breast cancer, a higher burden of physical symptoms has been associated with a reduced ability to pursue goals, and higher psychological distress [12]. Reducing physical symptoms or distress are common reasons that women with breast cancer seek complementary therapy [13]. Over the past decade, an approach known as integrative oncology, which integrates evidence-based complementary therapies and medical treatment and emphasizes patient-centered care and coordination between providers, has emerged [14]. The Ottawa Integrative Cancer Centre (OICC) practices integrative oncological care, treatment, and research. With community support, the OICC developed and operates the Head Start program, to address the distress expressed by many of their clients recently diagnosed with breast cancer. Funded by Babes4Breasts and free of charge to participants, Head Start offers education, resources, and skills to improve women's knowledge of integrative care options, reduce fear and anxiety, and strengthen their ability to cope with their diagnosis and incorporate life changes associated with living well with cancer.

A study of personal goals, which are expressions of life motivations, among women with breast cancer aligns with integrative oncology's focus on patient-centered care. In our literature search, we identified one published study of personal goals in integrative oncology [15]. Participants in an integrative oncology program in Vancouver, B.C. were asked to identify personal goals related to program participation. The personal goals identified highlighted important motivations related to breast cancer: improved well-being, increased chance of remission, increased physical energy, more effective pain management, and a return to active living [15]. But to design effective interventions that help women with breast cancer set and successfully pursue their goals, we first need to understand whether this population is able to pursue their goals and what factors may facilitate or obstruct that pursuit. In partnership with the OICC, we plan to examine the personal goals of women participating in Head Start in-depth. Our primary objective is to identify the perceived barriers to and facilitators of goal pursuit, including those related to Head Start. The secondary objectives are to describe the characteristics of participants' personal goals (goal content and dimensions) over time and to analyze whether women pursue their most important goals. Goal dimensions are factors which express how women think or feel about their goals (e.g., difficulty, control) [2]. They can be associated with important outcomes: higher goal importance may be associated with better psychological outcomes among people living with cancer, and higher goal stress with worse depressive mood and worse subjective health $[10,16]$. Towards their program objectives, Head Start works with women on mindfulness, awareness of emotions, identifying social supports, and use of coping mechanisms. Based on the aims and activities of Head Start, we hypothesize that there will be an increase in ratings of self-identity, perceived autonomy, and perceived support from others. In addition, we hypothesize a decrease in feelings of being scared and stressed over time among Head Start participants that will be greater than changes observed within the control group.

By publishing this study protocol, we provide transparency in our study plans and processes. It also enables external assessment of any changes between the protocol and the final study report, guarding against selective outcome reporting and other methodological changes influenced by study data. Finally, it strengthens the protocol by submitting it to the rigor of a peerreview process.

\section{METHODS}

\section{Study design}

This will be a prospective, observational cohort study of women participating in the OICC's Head Start program and, if available, controls taken from the program's waiting list ("wait-list controls").

\section{Participants and procedures}

Eligible individuals must be: either enrolled in one of two upcoming Head Start rounds (May and September 2017), or placed on a waiting list for either of these rounds; aged 18 years or older; female; and experiencing their first cancer diagnosis. They must also have good English comprehension and received a breast cancer diagnosis no more than five months prior to the start of the Head Start round for which they are enrolled or on the waiting list. Participants who are unable to provide informed consent will be excluded.

Recruitment will take place in the eight weeks prior to the first day of each Head Start round. Each Head Start round will last approximately five weeks. For each round, data will be collected at three time points: approximately one to fourteen days pri- 


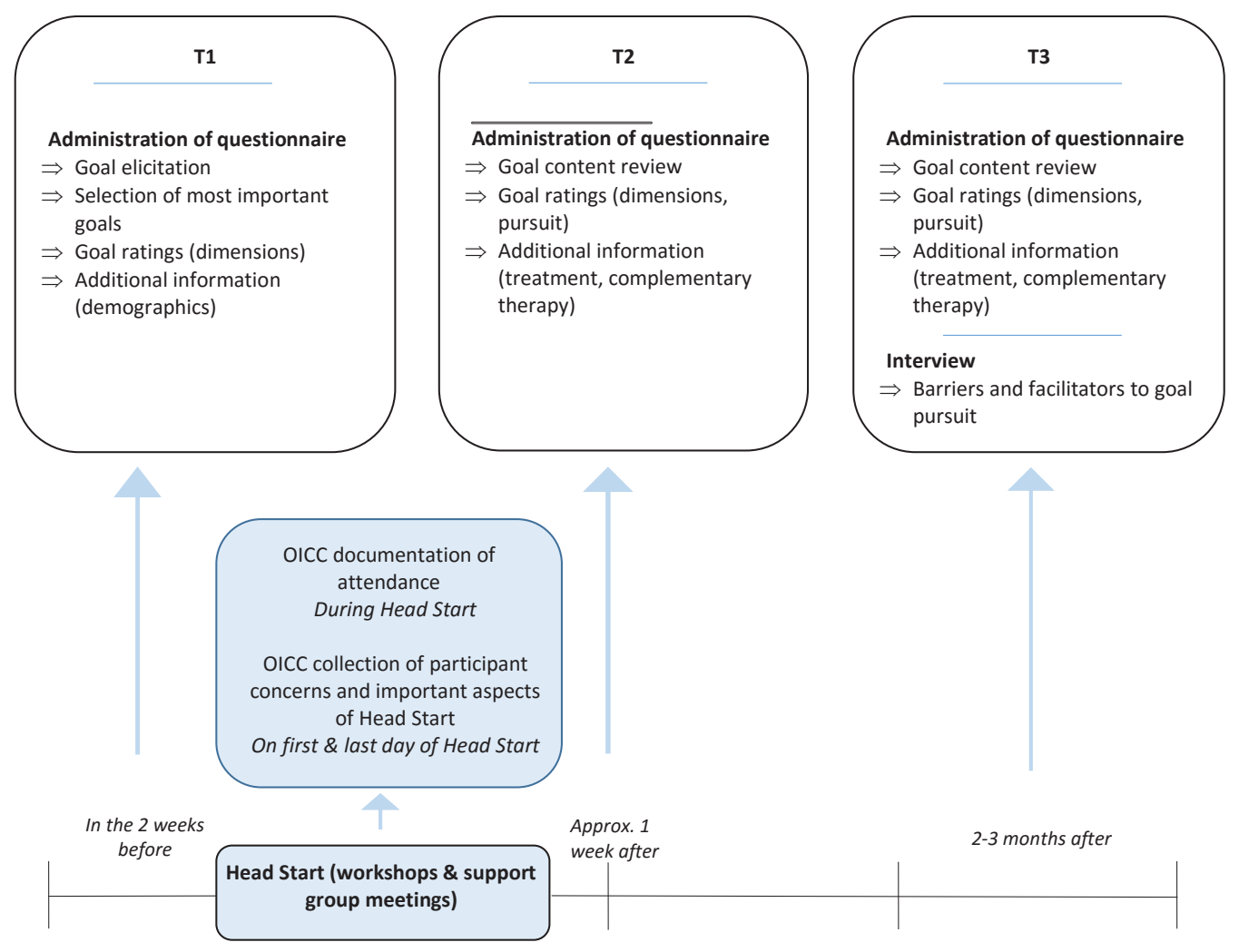

Figure 1. Illustration of study procedures. Illustration of study activities and timelines.

or to Head Start (T1 - baseline), approximately one week after Head Start ends (T2), and approximately two to three months after Head Start ends (T3). Study procedures are illustrated in Figure 1.

Ethics approval was obtained from the Research Ethics Boards of the Ottawa Hospital Science Network (OHSN-REB) and the Canadian College of Naturopathic Medicine. Participants will provide written informed consent consistent with the OHSNREB's guidelines before taking part in the study.

\section{Recruitment}

The OICC will conduct recruitment. Following OICC standard procedure, interested individuals will be assessed for eligibility for Head Start. Eligible women will enroll or be placed on a waiting list if maximum enrollment has been reached. If a woman also meets this study's eligibility criteria, the OICC will inform her of the study. Interested individuals will receive a letter of introduction about the study and the consent form. Study enrollment and consent will be conducted by the primary investigator (i.e. the first author). Recruitment started in May 2017.

\section{Sample size}

The anticipated sample size will be 18 to 36 , but will depend on the number of women interested in participating in selected Head Start rounds and the availability of a control cohort. This study is primarily concerned with examining the goals of Head Start participants, the "exposed" group. A control cohort will be included only if the following criteria can be met. If maximum enrollment (12 women) in each Head Start round is reached, the OICC places additional eligible individuals on a waiting list. If a minimum of 12 women on the waiting list enroll in this study, a control cohort will be formed. Based on our expectation of the Head Start enrollment process, the "exposed" group will have a maximum of 24 individuals ( 2 rounds of 12 individuals). 
Table 1. Data sources and measurement. Presentation of sources and measurements of data for each variable of interest.

\begin{tabular}{|c|c|c|}
\hline Variable of interest & $\begin{array}{l}\text { Source of } \\
\text { data }\end{array}$ & Method of measurement \\
\hline Participation in Head Start & $\begin{array}{l}\text { Head Start } \\
\text { attendance } \\
\text { records }\end{array}$ & $\begin{array}{l}\text { Yes/no. For "yes", number of workshop } \\
\text { days and number of support group } \\
\text { meetings attended will be counted }\end{array}$ \\
\hline $\begin{array}{l}\text { Barriers and facilitators to goal } \\
\text { pursuit, including Head Start } \\
\text { factors, cancer-related symptoms, } \\
\text { treatment factors, and } \\
\text { complementary therapy factors }\end{array}$ & $\begin{array}{l}\text { Participants, } \\
\text { Head Start } \\
\text { client records }\end{array}$ & $\begin{array}{l}\text { Semi-structured, face-to-face interviews } \\
\text { (participants); MYCAW data on important } \\
\text { treatment aspects (Head Start client } \\
\text { records) }\end{array}$ \\
\hline Personal goal elicitation & Participants & PPA-based goal elicitation \\
\hline Personal goal pursuit ratings & Participants & Ratings on a 5-point scale \\
\hline $\begin{array}{l}\text { Personal goal dimensions of: } \\
\text { challenge, likelihood of success, } \\
\text { autonomy, intention attention, } \\
\text { support, time adequacy, self- } \\
\text { identity, hopeful, scared, sad, } \\
\text { happy, and stressed }\end{array}$ & Participants & PPA-based goal ratings on a 11-point scale \\
\hline Demographic variables & Participants & $\begin{array}{l}\text { Participant assessment. Categorical: } \\
\text { current living situation, highest education } \\
\text { level completed, household income } \\
\text { (annual, Canadian dollars), breast cancer } \\
\text { stage. Continuous: age (years), time since } \\
\text { diagnosis (days) }\end{array}$ \\
\hline Conventional cancer treatment & Participants & $\begin{array}{l}\text { Participant assessment of whether } \\
\text { treatment is currently received }(y / n)\end{array}$ \\
\hline Complementary therapy & Participants & $\begin{array}{l}\text { Participant assessment of whether therapy } \\
\text { is currently received }(y / n)\end{array}$ \\
\hline
\end{tabular}

MYCAW: Measure Yourself Concerns and Well-Being questionnaire; PPA: Personal Projects Analysis

\section{DATA ELEMENTS AND INSTRUMENTS}

Data sources and assessment methods are summarized in Table 1. We will invite participants to complete paper-based questionnaires at T1, T2 and T3 (15 to 25 minutes to complete each). At T3, following completion of the questionnaire, participants will participate in a face-to-face, one-on-one interview (approximately 45 minutes). Interviews will be audio-recorded with participant consent.

\section{Head Start participation}

The exposure is Head Start participation: enrollment and attendance in a pre-specified Head Start round (yes/no). Nonparticipants will be on the waiting list to participate in Head Start, thus forming the wait-list control group for this study. If a spot becomes available in Head Start prior to the first day of the round and a wait-list control becomes a participant in Head 
Start, she will be included in the "exposed" cohort rather than the control group. Crossover is not possible after the program starts. We will count the days of Head Start participation in the exposed group using OICC attendance records linked to study records.

\section{Personal goals}

To identify personal goals, we will use the Personal Projects Analysis (PPA), a well-established methodological approach developed by Dr. Brian Little for collecting data on personal goals [5]. Personal goals are identified by the participant using his or her own phrasing, but goal dimensions are scored by standard means, allowing for comparison across participants [5]. The PPA allows for the assessment of multiple goals simultaneously and has been used to assess personal goals among a range of populations including people with cancer and people experiencing illness $[6,9,17-19]$. Consistent with the PPA, at $\mathrm{T} 1$, we will ask participants to list up to twelve personal goals, in their own words, over the next three to four months. Participants will then be asked to choose up to six goals among the twelve that are most important to them. At T2, participants will be shown their lists of six important goals from $\mathrm{T} 1$ and asked to annotate any changes they wish to make. They may remove or modify existing goals, add new ones, or leave the list as is. This process will be repeated at $\mathrm{T} 3$ with a review of the goals identified at $\mathrm{T} 2$.

\section{Personal goal dimensions}

Goal dimensions are "constructs on which goals vary" [2, p.340]. We will examine both cognitive and emotional dimensionshow people think and feel about their goals. Following selection of the six most important goals at $\mathrm{T} 1$, participants will rate each of their six goals (scale of 0 to 10) in various dimensions consistent with the PPA (challenge, likelihood of success, autonomy, intention, attention, support, time adequacy, selfidentity, hopeful, happy, sad, scared, stressed) $[5,20,21]$. This process will be repeated at $\mathrm{T} 2$ and $\mathrm{T} 3$, following goal content review. Participants will rate any new or modified goals identified at $\mathrm{T} 2$ as well, but will not rate any goals removed from the list at $\mathrm{T} 2$. This process will be repeated at $\mathrm{T} 3$ with a review of the goals identified at $\mathrm{T} 2$.

\section{Personal goal pursuit}

Goal pursuit is defined as actions taken to strive towards goals $[2,22]$. At T2, participants will assess their pursuit of each of the six important goals identified at $\mathrm{T} 1$ (including those removed at T2) by assigning a score on a five-point scale ( 1 'not at all' to
5 'as much as I could') to the question, "How much did you feel you pursued this project?". At T3, participants will repeat this process for the goals assessed at $\mathrm{T} 2$, along with any new goals identified at $\mathrm{T} 2$.

Barriers and facilitators of personal goal pursuit Participants will identify barriers and facilitators during faceto-face interviews at T3. Questions will be open-ended, but participants will also be asked specifically about the role, if any, of Head Start aspects, cancer-related symptoms, cancer treatment, and complementary therapies as barriers or facilitators to goal pursuit.

\section{Additional variables}

At T1, we will collect demographic data on: current living situation, highest education level completed, household income, breast cancer stage, age (years, measured at Head Start round start date), and time since diagnosis (days, measured to Head Start round start date). At T2 and T3, we will collect data on whether participants are currently receiving medical treatment for breast cancer, and whether participants are currently receiving complementary therapy (yes/no).

Data on important Head Start program elements will be collected from exposed participants only, using the Measure Yourself Concerns and Well-Being (MYCAW) questionnaire [23]. MYCAW was developed for use by cancer care services including complementary therapy centres to capture outcomes that are important to cancer patients. On the last day of Head Start (approximately one week before T2), the OICC will administer the MYCAW to participants. The MYCAW asks, "Reflecting on your time with the Head Start program, what were the most important aspects for you?"This data will be provided by the OICC to the investigator, with participant consent.

\section{PROTOCOL AMENDMENTS}

This protocol reflects amendments that were implemented after completion of the first version of the protocol (November 14, 2016), but prior to participant recruitment and data collection. Due to a delay in starting the study, we replaced an original plan to conduct feasibility testing with more intensive data collection tool development and will recruit participants from two rounds of Head Start instead of three. Protocol amendments are presented in Supplementary File 5. 


\section{DATA ANALYSIS}

\section{Qualitative analyses}

Barriers and facilitators to goal pursuit will be analyzed using inductive content analysis following Braun and Clarke's process guidelines [24]. Important aspects of Head Start from the MYCAW will be analyzed using the same thematic approach.

Goal content will be categorized by: (a) directional motivation (attain, maintain, or avoid); and (b) by themes using directed content analysis [25-27]. Preliminary thematic categories based on life domains suggested by Little will guide analysis, but final categories will be identified in an iterative process as data is analyzed [5].

\section{Quantitative analysis}

For the additional variables, we will calculate proportions (\%) for categorical variables. For continuous variables, mean and standard deviation (SD), or median and range if data is nonnormally distributed, will be used. We will use t-tests and chisquare tests to compare continuous and categorical data, respectively, between study groups (if a control group is formed), with $5 \%$ significance. If there are small cell counts, we will use an appropriate non-parametric test, such as the Wilcoxon Rank Sum Test, instead.

We will calculate descriptive statistics on personal goals, each goal dimension, and goal pursuit scores (number and mean/ SD or median/range, as appropriate), and on goal content (number and proportion of goals in each thematic category) within groups at each study timepoint. To analyze how personal goals change during the study, we will calculate the mean/SD (or median/range as appropriate) of the number of goals added, removed, and modified at T2 and at T3. We will use T-tests with $5 \%$ significance to compare the goal pursuit scores between groups (if a control group is formed).

\section{Sub-group analyses}

As this study is exploratory, we will conduct several descriptive sub-analyses to explore possible relationships with goal pursuit. We will calculate mean/SD (or median/range as appropriate) goal pursuit scores for: (a) those undergoing cancer treatment and those who are not at $\mathrm{T} 2$ and $\mathrm{T} 3$; (b) those receiving complementary therapy and those who are not at T2 and T3; (c) each goal content category; and (d) each goal dimension.

\section{Missing data}

The investigator will be nearby during questionnaire comple- tion to answer any questions that may otherwise lead to missing data. We will report the number of missing values for each variable of interest, the reasons for missing values (if known), and the number of missing data for each analysis. We will restrict quantitative analysis to individuals with complete data on variables required for a particular analysis.

\section{Loss to follow up}

Dropout may be influenced in some way by cancer (i.e., lack of time due to treatment, or lack of physical capacity due to symptoms), or be related to the exposure, as wait-list controls may feel less support to continue with the study. This study has been designed with a short timeframe to minimize loss to follow-up. Non-responsive participants will be contacted twice before being considered lost to the study.

At study end, we will assess the balance of dropout rates between groups. The analysis will include data collected before participants are lost to follow up. We will report the number of participants lost to follow-up. If differential dropout occurs between T1 and T2, and there are fewer than 12 control group members with data, we will focus on analysis within the exposed group only. If differential dropout occurs between T2 and T3, we will compare groups on goal pursuit at T2, but not at T3.

\section{DISCUSSION}

We have a unique opportunity to examine personal goals among women with breast cancer within five months of diagnosis, a critical time psychologically. Following a breast cancer diagnosis, anxiety, depressive symptoms, and emotional distress are common [28-31]. Weisman and Worden found that distress is highest two months into treatment [29].

We anticipate that study results will contribute new knowledge about the personal goal pursuits of women with breast cancer seeking integrative oncology care and the factors that facilitate or obstruct their goal pursuit. Recent studies suggest that people living with cancer adapt their personal goals as they adjust to treatment and survivorship, but the adaptation may be positive or negative. People living with cancer have demonstrated use of a range of strategies to adjust their goals, including aborting a goal and engaging in a new one and aborting a goal without engaging in a new one $[9,32,33]$. In a 2015 study, a minority of women, following their breast cancer surgery, coped with disruptions in their personal goal pursuit by not taking any actions to overcome goal interference; this response was associated with in- 
creased perception of goal unattainability over six months [34]. But positive goal adjustment may have important psychological effects in a cancer context: the ability to disengage from unattainable goals and engage in different goals is positively associated with well-being $[35,36]$. Understanding more about goal pursuit can inform interventions aiming to support the process of positive goal adjustment.

Our study limitations include a risk of attrition bias, where waitlist controls (if used) may be more likely to drop out than Head Start participants, as discussed. People who face more barriers to goal pursuit (for example, those are heavily affected physically or emotionally by their cancer diagnosis) may also face barriers to study participation, presenting a risk of response bias. The primary data elements will be collected via qualitative interviews and analyzed by a single unblinded researcher. To reduce the possibility of interviewer bias, we will use an interview guide, and interview questions have been reviewed for possible introduction of bias. The researcher will review every interview transcript for possible introduction of bias and adjust questions as needed to reduce bias. Other authors will also periodically review interview transcripts.

\section{CONCLUSION}

We anticipate that study findings will identify important information about the personal goals of women recently diagnosed with breast cancer. This information can inform the development of activities to support goal setting and pursuit in Head Start and other integrative oncology programs to enhance support to women recently diagnosed with breast cancer.

\section{REFERENCES}

1. Ryan RM, Deci EL. Self-determination theory and the facilitation of intrinsic motivation, social development, and well-being. Am Psychol. 2000;55(1):68-78.

2. Austin JT, Vancouver JB. Goal constructs in psychology: Structure, process, and content. Psychol Bull. 1996;120(3):338-75.

3. Elliot A, Thrash T. Achievement goals and the hierarchical model of achievement motivation. Educ Psychol Rev. 2001;13(2):139-56.

4. Emmons RA. Personal strivings: An approach to personality and subjective well-being. J Pers Soc Psychol. 1986;51(5):1058-68.

5. Little BR. Personal projects: a rationale and method for investigation. Environ Behav. 1983;15(3):273-309.

6. Little B. Generative Contexts of Personal Projects Analysis. In: Little BR, Philips SD, Salmela-Aro K, eds. Personal project pursuit: goals, action, and human flourishing. Mahwah, N.J.: Lawrence Erlbaum Associations; 2007. 3-49 p.

7. Palys TS, Little BR. Perceived life satisfaction and the organization of personal project systems. J Pers Soc Psychol. 1983:44(6):1221-30.

8. Locke EA, Latham GP. Building a practically useful theory of goal setting and task motivation: A 35-year odyssey. Am Psychol. 2002;57(9):705-17.

9. Peterman A, Lecci L. Personal projects in health and illness. In: Little BR,
Philips SD, Salmela-Aro K, eds. Personal project pursuit: goals, action, and human flourishing. Mahwah, N.J.: Lawrence Erlbaum Associations; 2007. 329-53 p.

10. Hullmann SE, Robb SL, Rand KL. Life goals in patients with cancer: a systematic review of the literature: Life goals in patients with cancer. Psychooncology. 2016;25(4):387-99.

11. Sulkers $E$, Janse $M$, Brinksma A et al. A longitudinal case-control study on goals in adolescents with cancer. Psychol Health. 2015;30(9):1075-87.

12. Stefanic N, Caputi P, Iverson DC. Investigating physical symptom burden and personal goal interference in early-stage breast cancer patients. Support Care Cancer. 2014;22(3):713-20.

13. Wanchai A, Armer JM, Stewart BR. Complementary and Alternative Medicine Use Among Women With Breast Cancer: A Systematic Review. Clin J Oncol Nurs. $2010 ; 14(4)$ :E45-E55.

14. Greenlee H, Balneaves LG, Carlson LE et al. Clinical practice guidelines on the use of integrative therapies as supportive care in patients treated for breast cancer. JNCI Monogr. 2014;2014(50):346-58.

15. Verhoef MJ, Mulkins A, Boon H. Integrative health care: how can we determine whether patients benefit? J Altern Complement Med. 2005;11(supplement 1):s-57-s-65.

16. Wallenius MA. Personal project content and stress: relations to subjective health and depressive mood. Soc Indic Res. 2007;81(1):35-50.

17. Little B, Gee T. The methodology of Personal Projects Analysis: four modules and a funnel. In: Little BR, Philips SD, Salmela-Aro K, eds. Personal project pursuit: goals, action, and human flourishing. Mahwah, N.J.: Lawrence Erlbaum Associations; 2007. 51-93 p.

18. Vroman K, Chamberlain K, Warner R. A Personal Projects Analysis: examining adaptation to low back pain. J Health Psychol. 2009;14(5):696-706.

19. Boersma SN, Maes S, Joekes K, Dusseldorp E. Goal processes in relation to goal attainment: predicting health-related quality of life in myocardial infarction patients. J Health Psychol. 2006;11(6):927-41.

20. Presseau J, Sniehotta FF, Francis JJ, Gebhardt WA. With a little help from my goals: Integrating intergoal facilitation with the theory of planned behaviour to predict physical activity. Br J Health Psychol. 2010;15(4):905-19.

21. Presseau J, Boyd E, Francis JJ, Sniehotta FF. Goal conflict and goal facilitation in community-based cardiac rehabilitation: A theory-based interview study. Psychol Health Med. 2015;20(2):227-38.

22. Gollwitzer PM, Brandstätter V. Implementation Intentions and Effective Goal Pursuit. J Pers Soc Psychol. 1997;73(1):186-99.

23. Paterson C, Thomas K, Manasse A, Cooke H, Peace G. Measure Yourself Concerns and Wellbeing (MYCaW): An individualised questionnaire for evaluating outcome in cancer support care that includes complementary therapies. Complement Ther Med. 2007;15(1):38-45.

24. Braun V, Clarke V. Using thematic analysis in psychology. Qual Res Psychol. 2006;3(2):77-101.

25. Elliot A, Friedman R. Approach-avoidance: a central characteristic of personal goals. In: Little BR, Philips SD, Salmela-Aro K, eds. Personal project pursuit: goals, action, and human flourishing. Mahwah, N.J.: Lawrence Erlbaum Associations; 2007. 97-118 p.

26. Wiese B. Successful Pursuit of Personal Goals and Subjective Well-Being In: Little BR, Philips SD, Salmela-Aro K, eds. Personal project pursuit: goals, action, and human flourishing. Mahwah, N.J.: Lawrence Erlbaum Associations; 2007. 301-25 p.

27. Hsieh H-F, Shannon SE. Three Approaches to Qualitative Content Analysis. Qual Health Res. 2005;15(9):1277-88.

28. Stanton AL, Wiley JF, Krull JL et al. Depressive episodes, symptoms, and trajectories in women recently diagnosed with breast cancer. Breast Cancer Res Treat. 2015;154(1):105-15.

29. Weisman $A D$, Worden JW. The existential plight in cancer: significance of the first 100 days. Psychiatry Med. 1976;7(1):1-15.

30. Zabora J, Brintzenhofeszoc K, Curbow B, Hooker C, Piantadosi S. The prevalence of psychological distress by cancer site. Psychooncology. 2001;10(1):19-28.

31. Henselmans I, Fleer J, de Vries J, Baas PC, Sanderman R, Ranchor AV. The adaptive effect of personal control when facing breast cancer: Cognitive 
and behavioural mediators. Psychol Health. $2010 ; 25(9): 1023-40$.

32. Pinquart M, Fröhlich C, Silbereisen RK. Testing models of change in life goals after a cancer diagnosis. J Loss Trauma. 2008;13(4):330-51.

33. Janse M, Fleer J, Smink A, Sprangers MAG, Ranchor AV. Which goal adjustment strategies do cancer patients use? A longitudinal study. Psychooncology. 2016;25(3):332-8.

34. Stefanic N, Caputi P, Lane L, Iverson DC. Exploring the nature of situational goal-based coping in early-stage breast cancer patients: a contextual ap proach. Eur J Oncol Nurs. 2015;19:604-11.

35. Wrosch C, Scheier MF, Miller GE, Schulz R, Carver CS. Adaptive self-regulation of unattainable goals: goal disengagement, goal reengagement, and subjective well-being. Pers Soc Psychol Bull. 2003;29(12):1494-508.

36. Schroevers M, Kraaij V, Garnefski N. How do cancer patients manage unattainable personal goals and regulate their emotions? $\mathrm{Br} J$ Health Psychol. 2008;13(3):551-62. 